\title{
The fusion of original and symmetric virtual images for image preprocessing in face recognition and collaborative representation based classification
}

\author{
Zhaojie Liu*Y Yirui Liu \\ Bio-Computing Research Center, Harbin Institute of Technology, HIT Campus of University Town of Shenzhen, Shenzhen, China
}

Received: March 21, 2017

Accepted: May 18, 2017

Online Published: June 1, 2017

DOI: $10.5430 / a i r . v 6 n 2 p 69$

URL: https://doi.org/10.5430/air.v6n2p69

\begin{abstract}
Various poses, facial expressions and illuminations are the biggest challenges in the fields of face recognition. To overcome these challenges, we propose a simple yet novel method in this paper by using the approximately symmetrical virtual face. Firstly, based on the symmetrical characteristics of faces, we present the method to generate the virtual faces for all samples in detail. Secondly, the collaborative representation based classification method is performed on both of the original set and virtual set individually. In this way, two kinds of representation residuals of every class can be obtained. Thirdly, an adaptive weighted fusion approach is presented and utilized to integrate those two kinds of representation residuals for face recognition. Lastly, we can obtain the label of the test sample by assigning it to the class with the minimum fused residual. Several experiments are conducted which show that the proposed method not only can greatly improve the classification accuracy, but also can effectively reduce the negative influence of various poses, illuminations, and facial expressions.
\end{abstract}

Key Words: Face recognition, Symmetric virtual face, Fusion scheme

\section{INTRODUCTION}

Face recognition is still an active research area in the fields of computation vision and pattern recognition owing to its huge commercial value and application prospect. ${ }^{[1-3]}$ In the past decades, various face recognition methods have been proposed, in which the representation based classification (RC) methods have attracted a great amount of attention owing to its good performance in reducing the negative influence of noises and occlusions. ${ }^{[4-10]}$ Sparse representation based classification (SRC), ${ }^{[1-14]}$ linear regression classification (LRC), ${ }^{[15]}$ and collaborative representation classification $(\mathrm{CRC})^{[16]}$ are the most well-known RC methods. These RC methods are all based on the assumption that each sample can be represented well by the joint linear combination of few samples from its own subspace and all use the representation residuals of every class for classification. The difference of these three methods is that LRC linearly represents the unknown samples by the combination of samples per class while SRC and CRC use all samples to represent the unknown sample and then produce corresponding linear combination vectors with different norm constraints, i.e., $1_{1}$ and $l_{2}$ norm constraints, respectively. Specially, for recognition of face with partial occlusion, such as wearing a scarf, glasses, etc., SRC can still maintain an outstanding recognition performance. ${ }^{[17]}$ However, SRC does not have any analytical solutions and is hard to directly solved due to the

\footnotetext{
*Correspondence: Zhaojie Liu; Email: 15773270726@163.com; Address: Bio-Computing Research Center, Harbin Institute of Technology, HIT Campus of University Town of Shenzhen, Shenzhen, China.
} 
constrain of $1_{1}$-norm. Fortunately, many fast and efficient methods have been proposed to solve the general model of SRC, such as the fast iterative shrinkage and thresholding algorithm (FISTA) ${ }^{[18]}$ and the 11-regularized least squares (L1LS), ${ }^{[19]}$ etc. In addition, based on the observation that the essential factor to achieve the highest accuracy of these RC methods is the collaborative representation rather than the sparse representation, various improved SRC methods have also proposed to further improve the classification accucary and efficiency. ${ }^{[20,21]}$

Although these SRC methods achieve very good performance in many cases, they still have some challenges, such as limited training samples, various expressions and illuminations, etc. $^{\text {[22-24] }}$ To this end, various improved methods have been proposed, in which the most simple but effective methods are the virtual sample based methods. ${ }^{[25-30]}$ Generally, for face recognition, the more training samples are used, the higher recognition accuracy will be. This is mainly because the face of a person can be better described by more samples. So generating some virtual but natural samples from the limited known samples is one of the reasonable and effective approaches to improve the face recognition accuracy. Virtual face images can be divided into two categories, i.e., twodimensional and three-dimensional virtual face images. ${ }^{[30]}$ Compared with the three-dimensional virtual face images, two-dimensional is much preferred in researches owing to its simplicity to obtain. Many schemes have been proposed to generate two-dimensional virtual face images. ${ }^{[20,31,32]}$ For example, considering that single face image is a wide range of uncertainty for representing the face, $\mathrm{Xu}$ et al. proposed to reduce this uncertainty by viewing the mean of two face images as virtual training samples. ${ }^{[27]}$ Ryu et al. ${ }^{[28]}$ exploited the distribution of the virtual training samples which are generated from the given training set. Liu et al. also represented every single image by some synthesized (shifted) samples. ${ }^{\text {[29] }}$ Based on the symmetry of faces, ${ }^{[33-36]}$ researchers proposed to generate the approximately symmetrical images as virtual samples for face recognition. ${ }^{[37]}$ Besides, Liu et al. ${ }^{[38]}$ demonstrates that using both of mirror face images and original face images not only can greatly improve the accuracy of face recognition, but also is effective to reduce the negative influence of various illuminations and poses. In Tang et $a l .,{ }^{[39]}$ several experiments are conducted to show the effectiveness in overcoming the problem of various appearances through using the virtual faces which generated from prototype faces. Su et al. ${ }^{[35]}$ also proved that using both of the original face images and their synthesized images can greatly improve the robustness of face detection.

The methods mentioned above show the available and reasonable in improving the classification accuracy through the combination of multiple images including both of original samples and virtual samples. In this paper, we propose another novel and simple method to solve the challenge problems of face recognition and improve the classification performance by synthesizing the virtual samples. We first present a simple method ${ }^{[40]}$ to generate the virtual face for each original face based on the symmetric property of face. Then CRC method is performed on the original set and virtual set, respectively. It should be noted that both virtual images and original image all contain discriminant and useful information for face recognition. Thus a better performance can be achieved by effectively exploited them. For this goal, we present an automatic and adaptive weighted fusion approach $^{[41]}$ to integrate the two kind of information. Finlay, the label of the test sample can be achieved by assigning it to the class with the minimum fused residual. We demonstrate it has great importance to distinguish an object from others. For convenience, we refer to the approximately symmetrical virtual face images presented in Xu et al. ${ }^{[40]}$ as "ASV" method. Compared with the ASV method, our method has the following merits. 1) The ASV scheme only uses original images or symmetrical virtual face images for image preprocessing. While for image preprocessing our method uses all of original images and symmetrical virtual face images. 2) The ASV method employs the nearest neighbour classifier for classification. However, we use sparse representation based classification algorithms, particularly the CRC method. 3) Compared with the ASV method, the scheme to fuse original and approximately symmetrical virtual samples has obtained a better performance on different datasets.

The remainder of the paper is organized as follows. Section 2 provides the proposed method. Section 3 offers s the analysis of our method. Our experiments and results are shown in Section 4. Section 5 provide our conclusion.

\section{THE DESCRIPTION OF THE PROPOSED METHOD}

In this section, we mainly present the proposed method in detail.

\subsection{The process of synthesizing an symmetrical virtual face image}

The ASV ${ }^{[40]}$ method can produce approximately symmetrical virtual face images automatically and has strong recognition capability. In this section, we summary it about how to generate a symmetric virtual face image in detail. Firstly, for an original face image, we can acquire the left vector and right vector as $z_{L}$ and $z_{R}$ respectively. Through different datasets, it can produce the approximately axis-symmetrical face images, which contains training and test samples. In particular, 
both $z_{L}$ and $z_{R}$ column vectors. The process of obtaining $z_{L}$ and $z_{R}$ are is as follows. Let $F$ be an original face image. Then $F_{j}(j=1,2, \cdots, J)$ denote that $j$-th column of 2D image matrix $F$. The pixel at the $i$-th row and $j$-th column of $F$ is denoted by $F_{i j}$. We suppose that $J$ is an even number. It is clear that $z_{L}$ is obtained by concatenating the first column to the $\frac{J}{2}$-th column of $F$ one by one and $z_{R}$ is obtained by concatenating the last column to the $\left(\frac{J}{2}+1\right)$-th column of $F$ one by one.

It should be pointed out that $z_{L}$ and $z_{R}$ are just initial values of original left-face image and original right-face image, respectively. In this subsection, we mainly present the approach to obtain approximately equal $z_{L}$ and $z_{R}$ by using the iterative gradient descent algorithm. By using the gradient descent algorithm, we can obtain the local optimal solution of $z_{L}$ and $z_{R}$. For $f(x)$, the gradient of $x_{n}$ is the fastest growing direction, then the opposite direction is the fastest direction to decline. The procedure of function $f(x)$ (gradient descent) is

$$
x^{n+1}=x^{n}-\eta \nabla f\left(x^{n}\right)
$$

where $\eta$ is the learning ratio, $x^{n+1}$ and $x^{n}$ are the values of $x$ at time $n+1$ and $n$, respectively. For the proposed method, we can define a reasonable function as $L\left(z_{L}, z_{R}\right)=$ $\left\|z_{L}-z_{R}\right\|_{2}^{2}$ When we continue to iterate, the final result will converge to a local minimum, till to that the $z_{L}$ and $z_{R}$ are approximately equal. For our experiments, obtain the minimum value of $L\left(z_{L}, z_{R}\right)$ is very important, it affects the efficiency of the algorithm. It is easy to get $\nabla_{z_{L}} L\left(z_{L}, z_{R}\right)=$ $2\left(z_{L}-z_{R}\right)$ and $\nabla z_{R} L\left(z_{L}, z_{R}\right)=2\left(z_{R}-z_{L}\right)$. As a result, the formulas to iteratively update $z_{L}$ and $z_{R}$ are

$$
\begin{aligned}
& z_{L}^{n+1}=z_{L}^{n}\left[1-\gamma\left(1-\left(\frac{z_{R}}{z_{L}}\right)^{n}\right)\right] \\
& z_{R}^{n+1}=z_{R}^{n}\left[1-\gamma\left(1-\left(\frac{z_{L}}{z_{R}}\right)^{n}\right)\right]
\end{aligned}
$$

In order to make the numerical stability, we set $\gamma$ to $\gamma=\frac{\eta}{n}$. The number of iterations is represented by $n$ and $\eta$ is a positive constant. When $n$ increases, the $\gamma$ will decrease, so it's convenient to obtain the local optimal solution. The iterative updating is stoped when the following two conditions are satisfied. 1) $\left\|z_{L}^{n+1}-z_{L}^{n}\right\|<\delta$, where $\delta$ stands for a very little positive constant and $z_{L}^{n}$ shows the value of $z_{L}$ at time $n$. 2) The predefined maximum value is more than or equal to the number of iterations. After we obtained the optimal vectors $z_{L}^{n}$ and $z_{R}^{n}$, the new synthesized symmetrical virtual image which has the same size as the original face image will be achieved through concatenating them.

\subsection{Related steps of the proposed method}

In this subsection, we mainly present the classification approach by integrating the original and virtual images. We first perform the CRC on the original dataset and virtual dataset individually. Then similar to traditional CRC method, two kinds of residuals, i.e., residual of the original test sample and original training samples per class, and that of residual in the virtual dataset, can be obtained. Suppose the dataset has $c$ subjects, every class has m samples. After we obtained the symmetrical virtual face images, every class has $2 \mathrm{~m}$ samples. Let $d_{a}^{i}(i=1, \cdots, c)$ be the dis-similarity between the original face images and test sample of the $i$-th subject. To integrate both of the original and virtual discriminant information, we propose an adaptively weighted fusion approach. Let $d_{b}^{i}(i=1, \cdots, c)$ be the dis-similarity between the symmetrical virtual face images and test sample of the $i$-th subject. Then $P_{a}^{1} \leq \cdots \leq P_{a}^{c}$ represents the sorted results of $d_{a}^{1,} \cdots, d_{a}^{c}$. Let $P_{b}^{1} \leq \cdots \leq P_{b}^{c}$ represents the sorted results of $d_{b}^{1,} \cdots, d_{b}^{c}$. We define $w_{10}=P_{a}^{2}-P_{a}^{1}$ and $w_{20}=P_{b}^{2}-P_{b}^{1}$. Then two adaptive weight is calculated as $w_{1}=\frac{w_{10}}{w_{10}+w_{20}}$ and $w_{2}=1-w_{1}$ for fusing the two residuals. The fused residual is obtained as

$$
\Delta_{i}=w_{1} d_{a}^{i}+w_{2} d_{b}^{i} \quad(i=1, \cdots, c)
$$

Where $\Delta_{i}$ stands for the new distance between a test sample and the $i$-th subject. Furthermore, we define the class label of the test sample as

$$
\lambda=\arg \min _{i} \Delta_{i}
$$

The main steps of our method can be described as follows.

Step 1: Apply the method of $\mathrm{ASV}^{[40]}$ to all original samples including training and test images to generate symmetrical virtual samples.

Step 2: CRC is performed on the virtual dataset and original dataset individually. Then two types of representation residuals of test sample and each class on the original and virtual datasets are achieved.

Step3: Using the proposed weighted fusion approach to adaptively integrate the discriminant information, i.e., representation residuals.

Step 4: Achieved the final result of the test sample by classifying it to the class with the minimum fused residual.

\section{THE ANALYSIS AND RATIONALES OF THE PROPOSED ALGORITHM}

In brief, the paper is the first work to fuse the original samples and approximately symmetrical virtual samples to perform 
face recognition. The following are the rationales of our method.

\subsection{The analysis of the proposed method}

In order to help the reader to understand the advantages of the proposed method more clearly, we define min-distance as follows. Let two-dimensional vector $E$ represent the test sample. The SRC method has used in this paper, suppose $X$ and $G_{r}$ represent the vector consisting of all the available training samples and the solution vector of the corresponding SRC, respectively. So we can let $d_{r}=\left\|E-X G_{r}\right\|$ denote the distance between the test samples and the $r$-th class. It is clear that the smaller the $G_{r}$ is, the greater effect of the $r$-th subject in the expression of the test sample is. In order to show the change before and after fusion of min-distance between the test samples and training samples, some figures are displayed. In our experiments, The YaleB ${ }^{[42]}$ and Georgia Tech $(\mathrm{GT})^{[43]}$ face databases were used to conduct our experiments. Figure 1 shows the min-distance between the test sample and training sample on the GT and YaleB face database, respectively. From the figure we can see that the after fused, the new min-distance in the between of original min-distance and virtual min-distance obtained from symmetrical virtual face images.
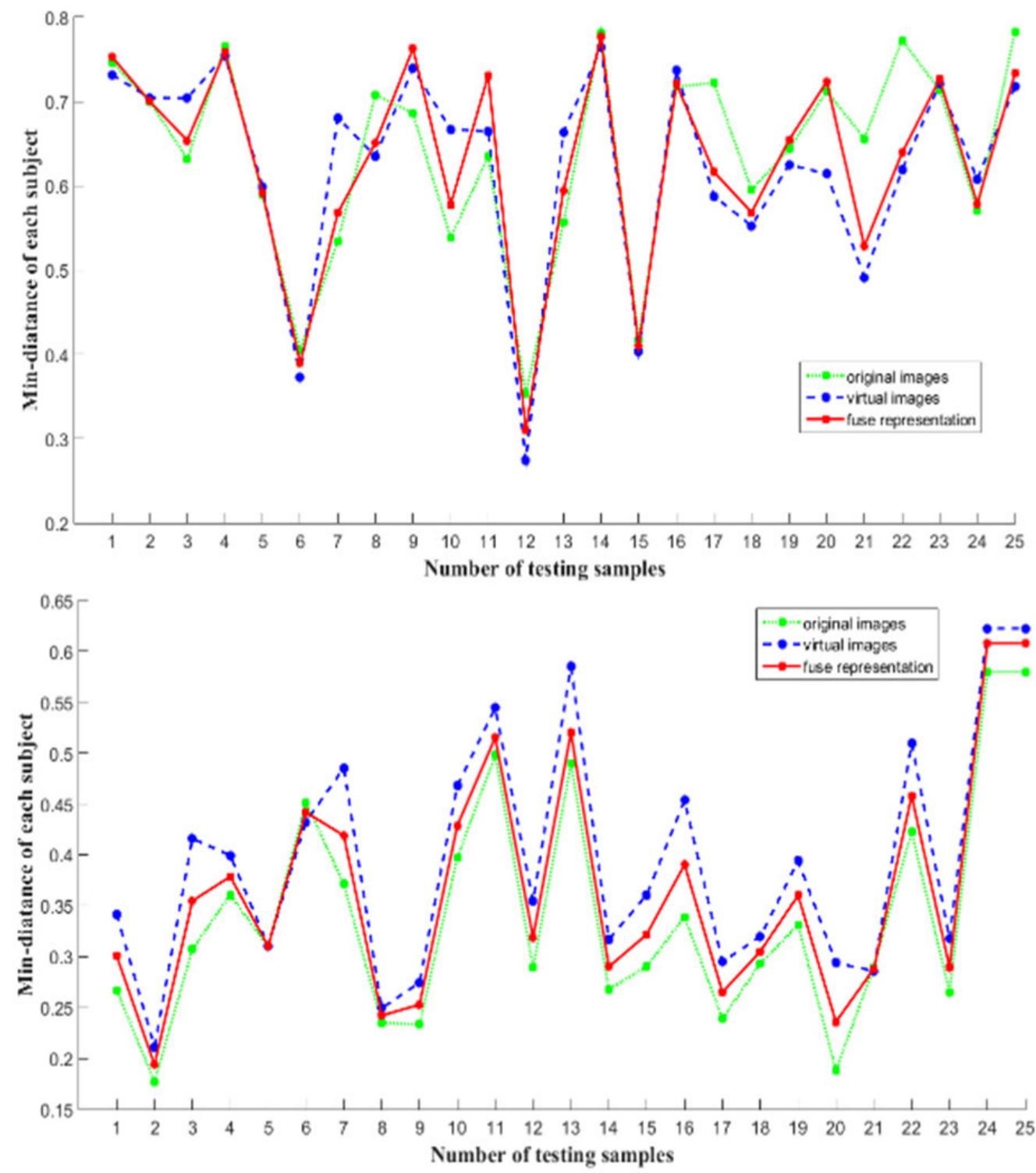

Figure 1. The minimum distance between the test sample and training sample on the GT and YaleB face databases, respectively. The green line depicts the minimum distance between the test sample and original training sample. The blue line depicts the minimum distance between the test sample and virtual training sample. The red line means the fused distance, which is obtained by using the proposed fusion approach. 


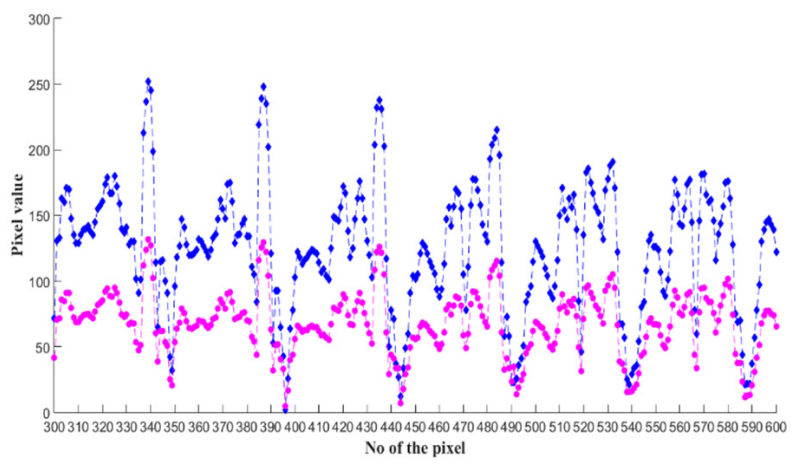

Figure 2. Pixel values of the 24-th sample of the first subject on the YaleB face database. The blue line depict the original pixel values. The magenta line depict the new pixel values which come from corresponding approximately symmetrical face image.

The process of producing approximately axis-symmetrical face image is so easy and simple to be implemented. Through the face detection procedure, when the face image in the real world is obtained during the register phase, its approximately symmetrical virtual face can be efficiently obtained by the method proposed in Xu et al. ${ }^{[40]}$ We provide the figures to show the difference between approximately symmetrical virtual images and its original images intuitively in this paper. The face vectors of left-half and right-half which from a same original face image are first acquired. And then we obtain the approximately symmetrical face image by the gradient descent algorithm and let the number of maximum iteration is 30 . Figure 2 shows the pixel values which generate from the symmetrical virtual face image and original face image of the YaleB database. To clearly show the obvious distinctions between symmetrical virtual face image and original face image, we select each image of the 300th to 600th pixel values of each image. From the figure we see that there is an obvious differences between the original image and symmetrical virtual image. Especially the side illumination is stronger, the difference is greater.

Therefore, in our proposed method, it is very reasonable to fuse the representation performances of original dataset and virtual dataset since both of them contain discriminant information for classification. Figure 3 shows the original pixel values from the left-face and right-face images of the 24-th sample of the first subject on the YaleB face database. Figure 4 shows the pixel values of the left-face and the right-face from the corresponding approximately symmetrical virtual sample in Figure 2. From the above two figures we see that there is a big difference between the left-face and right-face of the original sample under the condition of imbalance illumination. However, in the corresponding symmetrical virtual sample, the left-face is similar to the right-face. This also illustrates the fact that symmetrical virtual face image of Published by Sciedu Press our proposed method is indeed approximately axi-symmetric face image.

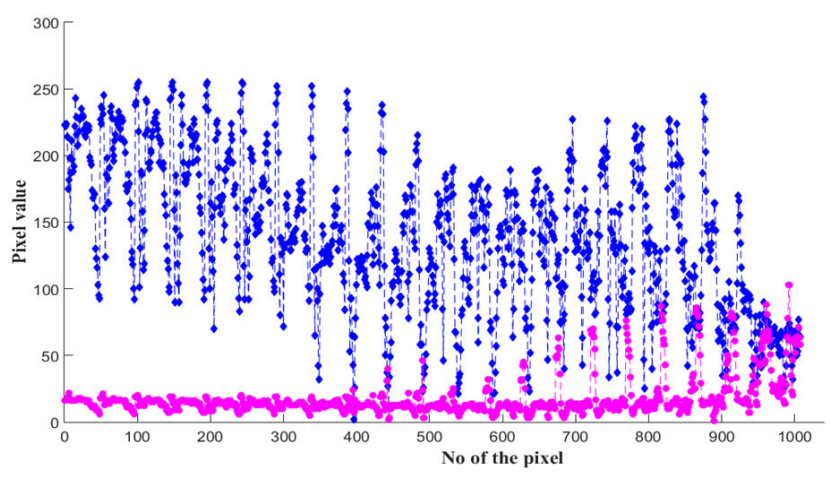

Figure 3. Pixel values of the left-face and the right-face from the original twenty-four sample of the first subject on the YaleB face database. The blue line depict the left-face pixel values. The magenta line depict the reversed right-face pixel values.

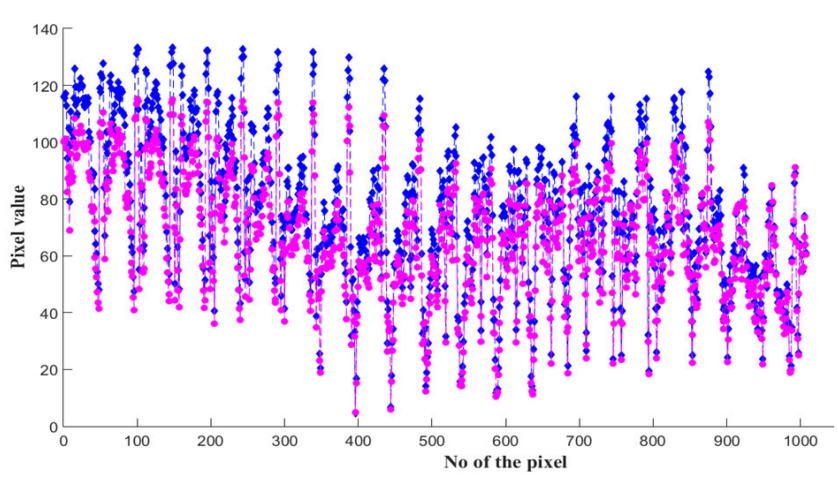

Figure 4. Pixel values of the left-face and the right-face from the corresponding approximately symmetrical sample in Figure 2. The blue line depict the left-face pixel values. The magenta line depict the reversed right-face pixel values.

\subsection{Advantages of the proposed method}

The YaleB face database was obtained with varying illuminations and different facial expressions. Figure 5 shows some original face images and corresponding symmetrical virtual images which generated from original face images on the YaleB face database.

The Georgia Tech (GT) face database contains 50 people of images and each person has 15 samples. Figure 6 shows several approximately axis-symmetrical generated from original face images of the GT face database and the corresponding original face images of a subject. From these figures we see that a better face image quality can be ontained from our method, and mitigate the negative influence of unbalanced illumination. 

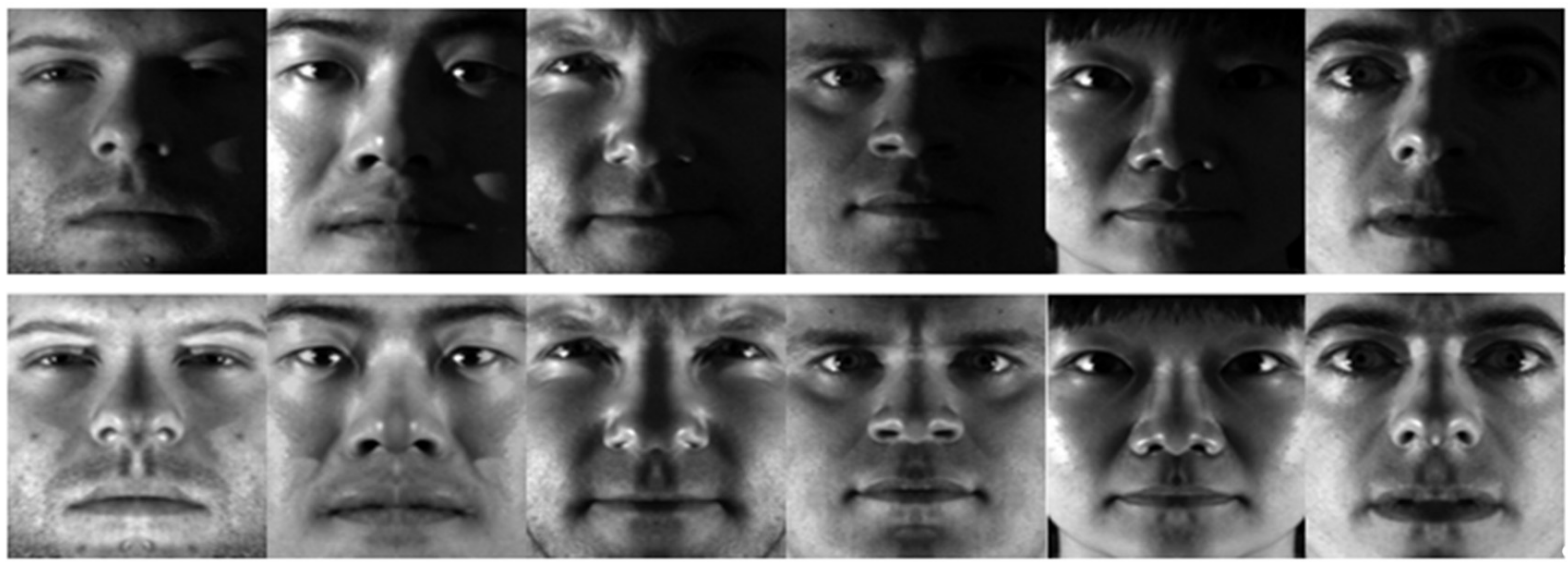

Figure 5. Some original samples from the YaleB face database and the corresponding approximately symmetrical samples. The first row shows the original samples. The second row show the approximately symmetrical samples generated from the original sample.

The SRC method which merely exploits the original images, however, the proposed method uses the original images and the approximately symmetrical virtual images simultaneously so the test sample may be better to represented, which encourages the method to obtain a better performance than those of the conventional methods. The proposed method first represents the test sample by original samples and virtual samples individually. In this way, two representation residuals of each class, i.e., representation residuals of original samples and virtual samples, are obtained. Then a weighted fusion approach is utilized to integrate the two residuals for image classification, in which the sample is classified to the class with the minimum fused residual.
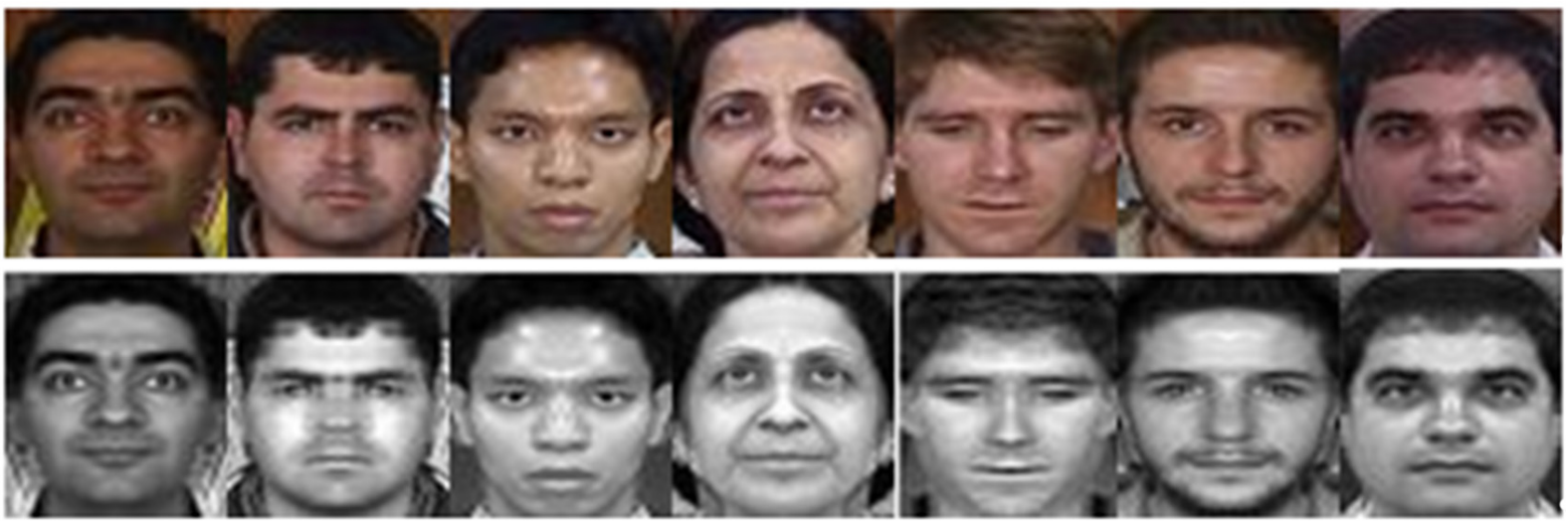

Figure 6. Some original samples from the GT face database and the corresponding approximately symmetrical samples. The first row shows the original samples. The second row show the approximately symmetrical samples generated from the original sample.

Our proposed method has following advantages. Firstly, the simultaneously use of the symmetrical virtual and original sample allows we exploit more complementary information which encourages the method to obtain a better classification accuracy. Secondly, our fusion scheme about the original image and approximately symmetrical virtual images can decrease the side effects of the illumination and pose difference. Thirdly, our fusion scheme is able to as an image preprocessing way to all training images and test images from different datasets.
The next section about experimental results show that the fusion of original image and symmetrical virtual image is indeed helpful and beneficial to obtain a better performance.

\section{EXPERIMENTAL RESULTS}

We make full use of original image and symmetrical virtual image to exploit their complementary information and evaluate the performance of our method by comparing with other face recognition methods. The aim of experiments presented in this section is to show it is appropriate to ap- 
ply the collaborative representation algorithm to consolidate the symmetrical virtual and original images to classify the images effectively. Besides the CRC of our method, we also use the FISTA algorithm to perform classification. The proposed method performs collaborative representation and FISTA, respectively. The two algorithms mentioned above are applied to the original and symmetrical virtual face sample for classification. We experimentally compare our fusion algorithm with the approximately symmetrical virtual face images which proposed in Xu et al. ${ }^{[40]}$ for face classification. The corresponding comparison experiment is implemented by just replacing the fusion algorithm of our method with nearest neighbour classifier.

In order to show advantage of using the approximately symmetrical face image of our method, we also compare it with mirror image ${ }^{[42]}$ by the same fusion algorithm. When nearest neighbor classifier is used as compared method, we refer to it as "Native symmetrical + NN". When we use the collab- orative representation and FISTA in our method, we refer to it as "our method + collaborative representation", "our method + FISTA" respectively. When the collaborative representation and FISTA are applied to only the original images, we refer to it as "the native CR (collaborative representation)", "the native FISTA" respectively. When we use the mirror image to replace the approximately axis-symmetrical image in our method, we refer to it as "mirror + collaborative representation". In addition, in order to show the rationality of obtaining the distance between symmetrical virtual testing sample and symmetrical virtual sample, we use the distance from the original testing sample and symmetrical virtual training sample to replace it. We refer to it as "the changed testing sample + collaborative representation".

We used the GT, ${ }^{[43]}$ Near infrared (NIR), ${ }^{[44]}$ Yale $^{[45]}$ and YaleB face databases to evaluate the proposed method. Tables 1, 2 and 3 show the rate of classification errors on the NIR, Yale and YaleB respectively.

Table 1. The rate of classification errors (\%) on NIR face database

\begin{tabular}{lll}
\hline Training simple per class & $\mathbf{2}$ & $\mathbf{3}$ \\
\hline Our method + collaborative representation & $\mathbf{4 . 0 0 0}$ & $\mathbf{8 . 0 0 0}$ \\
Native symmetrical + NN & 7.000 & 13.00 \\
The changed testing simple + collaborative representation & 5.500 & 10.00 \\
Our method + FISTA & 9.500 & 12.00 \\
The native CR & 4.000 & 9.00 \\
The native FISTA & 8.500 & 12.00 \\
Mirror + collaborative representation & 6.000 & 11.00 \\
\hline
\end{tabular}
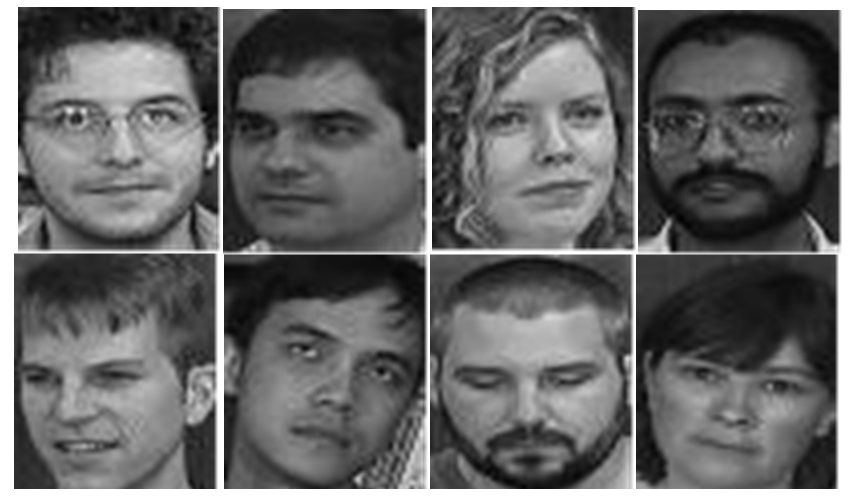

Figure 7. Some test samples on the GT database that were correctly and erroneously classified by our method and LWT, respectively.

\subsection{Experiment on the GT face database}

In our experiments, we evaluate the capability of our scheme in image preprocessing on the GT database. We compared it with the logarithmic wavelet transform (LWT) ${ }^{[46]}$ method.
The first 2 and 3 images of each subject were taken as training samples and the remaining of each subject were treated as test samples, and we use the nearest neighborhood (NN) classifier to obtain the final classification results. The experimental results in Figure 7. show that some test samples that were correctly and erroneously classified by our method and LWT, respectively.

\subsection{Experiment on the NIR face database}

We adopt the NIR ${ }^{[44]}$ database which includes 400 samples taken from 100 subjects, every individual providing 4 face samples. Images of the NIR face contain good facial detail for face recognition. Each of the face images contains pixels. Figure 8 shows some image examples of four subjects in NIR database. For each subject, the 2 and 3 face images were used as training samples and the remaining were used as test samples. Table 1 shows the experimental results of our method and other classification method. It proves that the proposed scheme can obtain a good performance once again. 

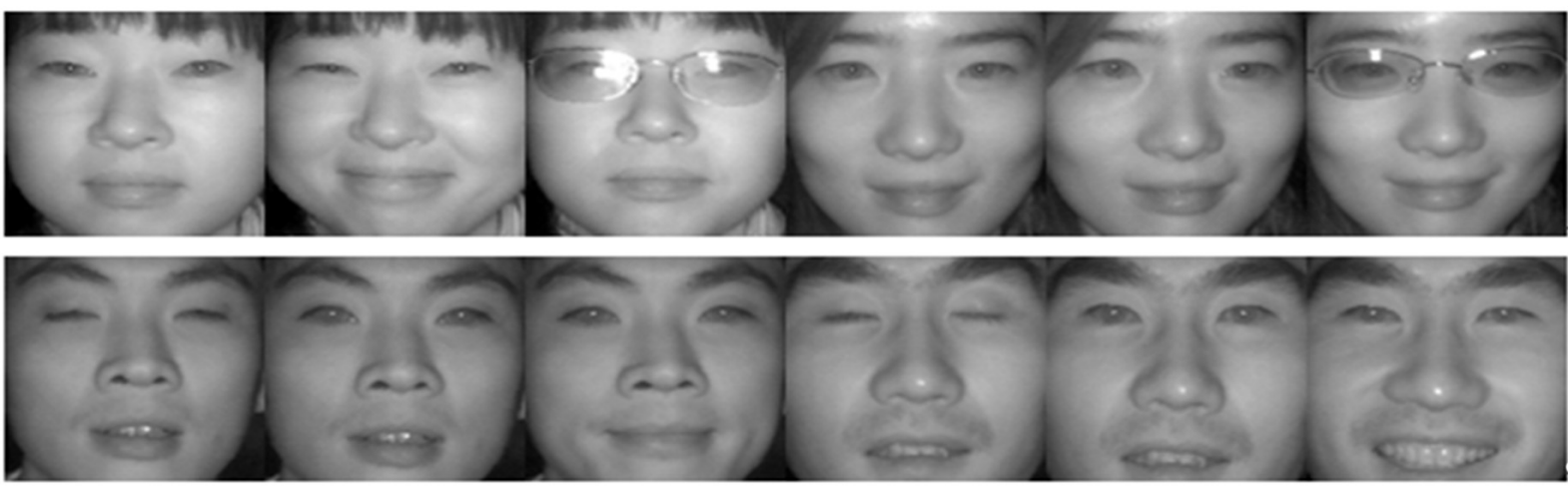

Figure 8. Part of image examples of two subjects in the NIR dataset

\subsection{Experiment on the Yale face database}

We adopt the Yale database which consists of 15 individuals and every individual providing 11 face samples. Under different lighting conditions, the 480 face samples contains many different facial expressions such as winking, surprised, happy, sleepy, sad and normal. In addition, a few face im- ages even wear glasses. Each of these face images was also resized to 100 pixels $\times 100$ pixels. For each subject, the first 1, 2, 3 and 4 face images were used as training samples and the remaining were used as test samples respectively. Figure 9 shows some image examples of two subjects in the Yale database.

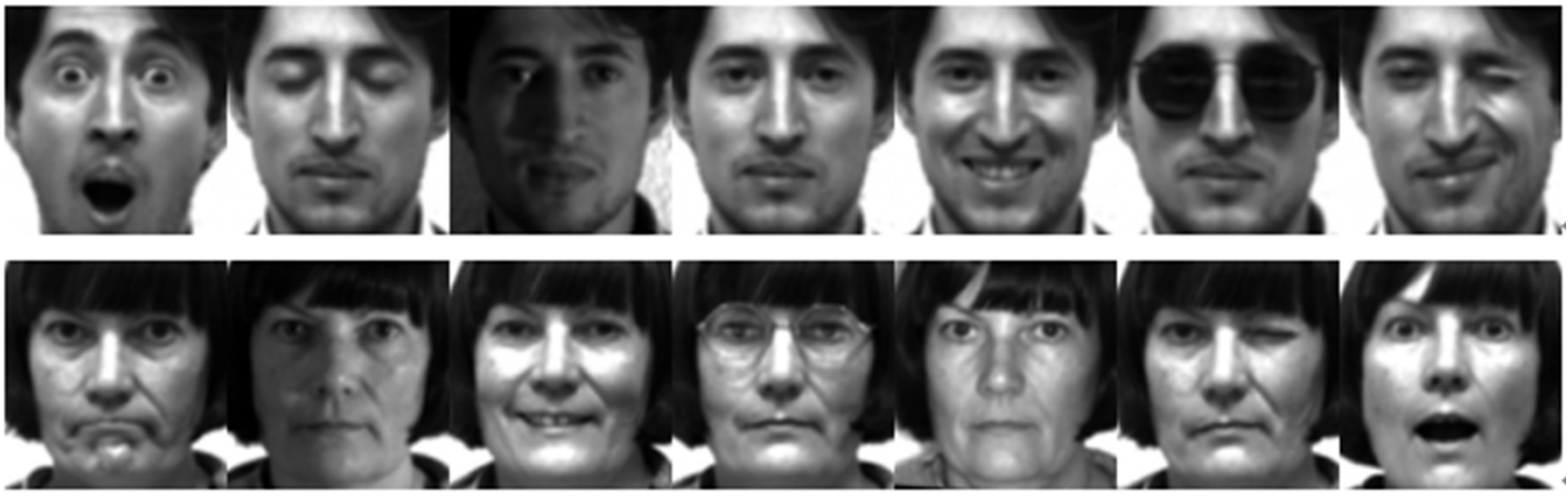

Figure 9. Part of image examples of two subjects in the Yale dataset

Table 2. The rate of classification errors (\%) on Yale face database

\begin{tabular}{lllll}
\hline Training simple per class & $\mathbf{1}$ & $\mathbf{2}$ & $\mathbf{3}$ & $\mathbf{4}$ \\
\hline Our method + collaborative representation & $\mathbf{2 3 . 3 3}$ & $\mathbf{7 . 4 1 0}$ & $\mathbf{0 . 8 3 0}$ & $\mathbf{1 . 9 0 0}$ \\
Native symmetrical + NN & 24.00 & 7.410 & 5.000 & 4.760 \\
The changed testing simple + collaborative representation & 26.67 & 11.11 & 5.000 & 4.760 \\
Our method + FISTA & 38.00 & 10.37 & 8.330 & 3.810 \\
The native CR & 31.94 & 16.56 & 10.83 & 2.860 \\
The native FISTA & 18.00 & 20.74 & 19.17 & 7.620 \\
Mirror + collaborative representation & 33.06 & 19.69 & 18.57 & 13.00 \\
\hline
\end{tabular}

Table 2 shows the experimental results of our method and other classification method. We can learn that compared with other methods, ours method indeed gets a lower error rate of classification. For instance, the training samples of all samples separated from the rest of the test samples, the first three face images of each subject were designed as for- mer, the remaining images were used as latter, the error rates of classification of ours method, the changed testing sample +collaborative representation, our method + FISTA, the native $\mathrm{CR}$, the native FISTA, and mirror +collaborative representation method are $0.830 \%, 5.000 \%, 8.330 \%, 10.83 \%$, $19.17 \%$ and $18.57 \%$, respectively. 


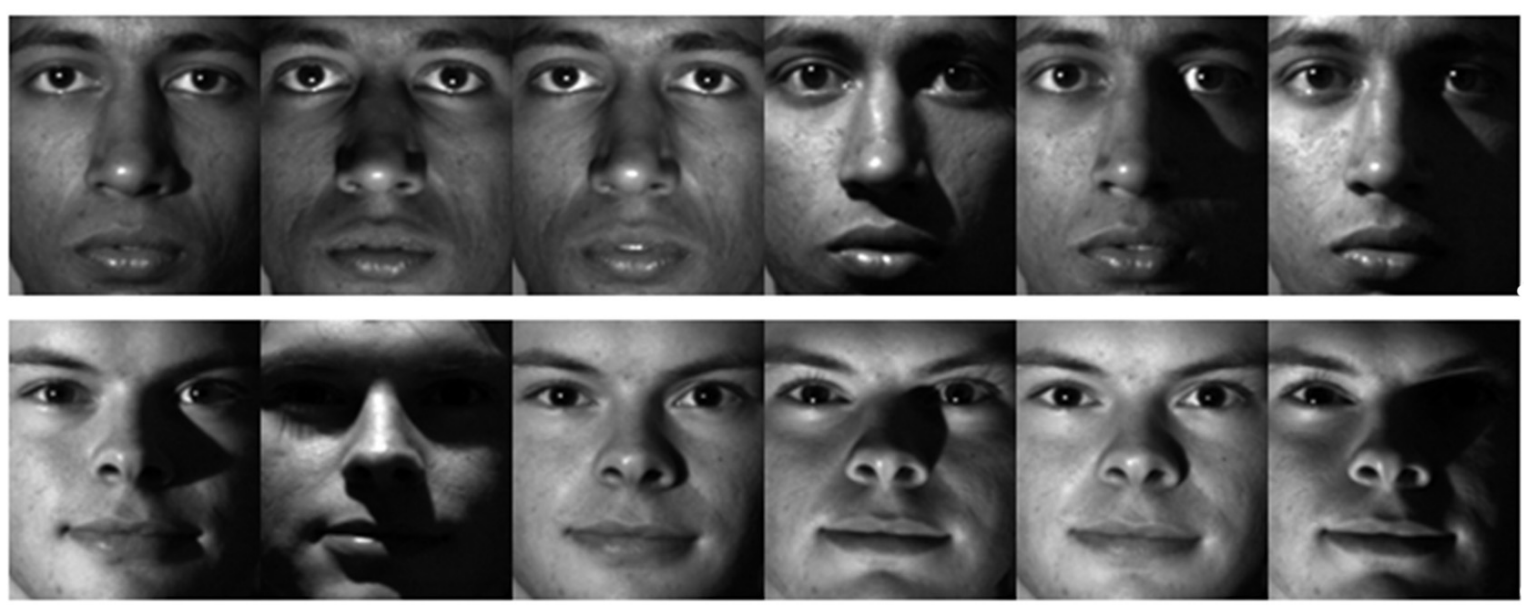

Figure 10. Part of image examples of two subjects in the YaleB dataset

\subsection{Experiment on the YaleB face database}

We also evaluate the capability of the proposed scheme on the YaleB face database in this subsection. There are totally 38 subjects with different facial expressions, in varying lighting conditions, each subject has 64 samples in the YaleB database. The size of every original face images is 192 pixels $\times 168$ pixels. The YaleB face database is a large-scale database. In order to the effect of our experiment, we select 15 individuals and every individual has 32 samples, which contains 480 samples totally. Each of these original face images was resized to 96 pixels $\times 84$ pixels. Some sample examples of two subjects in the YaleB database were shown in Figure 10. The original training samples set include the first 1,2, 3,5, and 6 samples of each subject, and the remaining face samples as the test samples. The Table 3 shows the rate of error classification of our method and other classification method in the YaleB database. At last, the experimental results prove that our proposed method still performs well as before when compared with other schemes.

Table 3. The rate of classification errors $(\%)$ on YaleB face database

\begin{tabular}{lllllll}
\hline Training simple per class & $\mathbf{1}$ & $\mathbf{2}$ & $\mathbf{3}$ & $\mathbf{4}$ & $\mathbf{5}$ & $\mathbf{6}$ \\
\hline Our method + collaborative representation & $\mathbf{3 5 . 4 8}$ & $\mathbf{2 6 . 8 9}$ & $\mathbf{2 5 . 0 6}$ & $\mathbf{1 7 . 6 2}$ & $\mathbf{1 7 . 5 3}$ & $\mathbf{1 8 . 2 1}$ \\
Native symmetrical + NN & 40.65 & 32.89 & 27.13 & 26.43 & 19.26 & 18.72 \\
The changed testing simple + collaborative representation & 36.34 & 28.89 & 25.06 & 19.05 & 19.01 & 21.79 \\
The native CR & 38.28 & 32.00 & 31.95 & 32.62 & 30.62 & 33.85 \\
The native FISTA & 38.06 & 29.11 & 27.86 & 26.67 & 35.31 & 37.44 \\
Mirror + collaborative representation & 38.28 & 31.78 & 29.43 & 18.81 & 20.00 & 23.33 \\
\hline
\end{tabular}

\section{Conclusion}

This paper clearly demonstrates that our fusion scheme about the original image and approximately symmetrical virtual images is able to decrease the side effects of the pose difference and illumination between the training and testing samples of the same face. Besides, it can be exploited to reflect some possible variation of the face images. The process of generating approximately symmetrical samples is an automatic process. The analysis and experimental results on several different face databases sufficiently show that our method is very simple and computationally very efficient.

\section{REFERENCES}

[1] Liu C, Yang J, Huang H. P-SURF: A robust local image descriptor. Journal of Information Science \& Engineering. 2011; 27(6): 2001-15.

[2] Hong X, Zhao G, Pietikainen M, et al. Combining lbp difference and feature correlation for texture description. Image Processing IEEE Transactions on. 2014; 23(6): 2557-68. PMid:24733014. https://doi.org/10.1109/TIP.2014.2316640
[3] Ma Z, Wen J, Liu Q, et al. Near-infrared and visible light image fusion algorithm for face recognition. Journal of Modern Optics. 2015; 62(9): 745-53. https://doi.org/10.1080/09500340.2 015.1005187

[4] Wright J, Ma Y, Mairal J, et al. Sparse representation for computer vision and pattern recognition. Proceedings of the IEEE. 2010; 98(6): 1031-44. https ://doi.org/10.1109/JPROC . 2010. 2044470 
[5] Wright J, Yang A, Ganesh A, et al. Robust face recognition via sparse representation. IEEE Trans. Pattern Anal. Mach. Intell. 2009; 31 (2) 210-27. PMid:19110489. https : //doi .org/10 . 1109/TPAMI . 20 08.79

[6] Yang J, Wright J, Huang T, et al. Image super-resolution via sparse representation. IEEE Trans. Image Process. 2010; 19 (11): 2861-73. PMid:20483687. https ://doi.org/10.1109/TIP.2010.20506 25

[7] Asif MS, Romberg J. Dynamic updating for L1 minimization. IEEE Journal of Selected Topics in Signal Processing. 2009; 4(2): 421-34. https ://doi.org/10.1109/JSTSP. 2009. 2039174

[8] Han B, Wu F, Wu D. Image representation by compressive sensing for visual sensor networks. J. Vis. Commun. Image Represent. 2010; 21 (4): 325-33. https://doi .org/10.1016/j.jvcir.2010.02. 007

[9] Fan Z, Ni M, Zhu Q, et al. L0-norm sparse representation based on modified genetic algorithm for face recognition. J. Vis. Commun. Image Represent. 2015; 28 (C): 15-20. https ://doi .org/10.101 6/j.jvcir.2015.01.001

[10] Lu CY, Min H, Gui J. Face recognition via weighted sparse representation. J. Vis. Commun. Image Represent. 2013; 24 (2): 111-6. https://doi.org/10.1016/j.jvcir.2012.05.003

[11] Zhang Z, Xu Y, Yang J, et al. A survey of sparse representation: algorithms and applications. IEEE Access. 2015; 3: 490-530. https://doi.org/10.1109/ACCESS . 2015. 2430359

[12] Cheng H, Liu Z, Yang L, et al. Sparse representation and learning in visual recognition: Theory and applications. Signal Process. 2013; 93 (6): 1408-25. https ://doi.org/10.1016/j.sigpro. 2012. 09.011

[13] Schmidt M, Fung G, Rosales R. Optimization methods for 11regularization. University of British Columbia. Technical Report TR-2009-19; 2009.

[14] Tropp JA, Gilbert AC, Strauss MJ. Algorithms for simultaneous sparse approximation. Part I: Greedy pursuit. Signal Process. 2006; 86 (3): 572-88. https://doi.org/10.1016/j.sigpro. 2005 05.030

[15] Naseem I, Togneri R, Bennamoun M. Robust regression for face recognition. Pattern Recognit. 2012; 45 (1): 104-18. https : //doi . org/10.1016/j.patcog.2011.07.003

[16] Zhang L, Yang M, Feng X. Sparse representation or collaborative representation: which helps face recognition? In: Proceedings of IEEE International Conference on Computer Vision. 2011: 471-8. https://doi.org/10.1109/iccv.2011.6126277

[17] Min R, Hadid A, Dugelay JL. Efficient detection of occlusion prior to robust face recognition. The Scientific World Journal. 2014; 2014(3): 519158. https ://doi .org/10.1155/2014/519158

[18] Gong PH, Zhang CS, Lu ZS, et al. A general iterative shrinkage and thresholding algorithm for non-convex regularized optimization problems. In: Proceedings of International Conference on Machine Learning, ICML. 2013: 37-45.

[19] Boyd SP; 2008 (accessed 04.08). http://web. stanford.edu/ b oyd/11_1s/

[20] Song X, Feng Z, Hu G, et al. Half-Face Dictionary Integration for Representation-Based Classification. IEEE Transactions on Cybernetics. 2015; 47(1): 142-52. https ://doi.org/10.1109/TCYB. 2 015.2508645

[21] Xu Y, Zhu Q, Fan Z, et al. Using the Idea of the Sparse Representation to Perform Coarse-to-Fine Face Recognition. Information Sciences. 2013; 238(20): 138-48. https://doi.org/10.1016/j.ins. 20 13.02 .051

[22] Mansano A, Matsuoka JA, Afonso LCS, et al. Improving image classification through descriptor combination. In: Proceedings of
SIBGRAPI. 2012; 2003: 324-9. https ://doi.org/10.1109/si bgrapi.2012.52

[23] Wen X, Wen J. Improved the minimum squared error algorithm for face recognition by integrating original face images and the mirror images. Optik-International Journal for Light and Electron Optics. 2016; 127(2): 883-9. https://doi.org/10.1016/j.ijleo.20 15.10 .182

[24] Ho HT, Chellappa R. Pose-invariant face recognition using Markov random fields. IEEE Trans. Image Process. 2013; 22:1573-84. PMid:23247858. https ://doi.org/10.1109/TIP. 2012.22334 89

[25] Thian NPH, Marcel S, Bengio S. Improving face authentication using virtual samples. In: Proceeding of the IEEE International Conference on Acoustics, Speech, and Signal Processing. 2003: 6-10. https://doi.org/10.1109/icassp.2003.1199150

[26] Sharma A, Dubey A, Tripathi P, et al. Pose invariant virtual classifiers from single training image using novel hybrid-eigenfaces. Neurocomputing. 2010; 73(10-12): 1868-80. https://doi.org/10.1016/ j.neucom.2009.10.027

[27] Xu Y, Fang X, Li X, et al. Data uncertainty in face recognition. IEEE Transactions on Cybernetics. 2014; 44(10): 1950-61. PMid:25222733. https ://doi.org/10.1109/TCYB.2014.2300 175

[28] Ryu YS, Oh SY. Simple hybrid classifier for face recognition with adaptively generated virtual data. Pattern Recognition Letters. 2002; 23 (7): 833-41. https : //doi.org/10.1016/S0167-8655(01)0 0159-3

[29] Liu J, Chen S, Zhou ZH, et al. Single image subspace for face recognition. In: Proceedings of the 3rd International Conferenceon Analysis and Modeling of Faces and Gestures. 2007: 205-19. https: //doi.org/10.1007/978-3-540-75690-3_16

[30] Hu Y, Jiang D, Yan S, et al. Automatic 3D Reconstruction for Face Recognition. FGR. 2004: 843-50.

[31] Xu Y, Zhang B, Zhong Z. Multiple representations and sparse representation for image classification. Pattern Recognition Letters 2015; 68: 9-14. https://doi.org/10.1016/j.patrec. 2015. 07.032

[32] Xu Y, Li X, Yang J, et al. Integrating conventional and inverse representation for face recognition. IEEE Transactions on Cybernetics. 2014; 44(10): 1738-46. PMid:25222718. https ://doi.org/10.1 109/ТCYB. 2013. 2293391

[33] Xu Y, Zhu X, Li Z, et al. Using the original and'symmetrical face' training samples to perform representation based two-step face recognition. Pattern Recognit. 2013; 46 (4): 1151-8. https: //doi.org/10.1016/j.patcog.2012.11.003

[34] Saha S, Bandyopadhyay S. A symmetry based face detection technique. In: Proceedings of the IEEE WIE National Symposium on Emerging Technologies. 2007: 1-4.

[35] Su MC, Chou CH. Application of associative memory in human face detection. In: Proceedings of the International Joint Conference on Neural Networks. 1999; 5: 3194-7. https://doi.org/10.1109/ IJCNN . 1999.836165

[36] Saber E, Murat-Tekalp A. Frontal-view face detection and facial feature extraction using color, shape and symmetry based cost functions. Pattern Recognition Letters. 1998; 19 (8): 669-80. https : //doi.org/10.1016/S0167-8655(98)00044-0

[37] Song X, Yang X, Shao C. Parity symmetrical collaborative representation-based classification for face recognition. International Journal of Machine Learning \& Cybernetics. 2016:1-8. https: //doi.org/10.1007/s13042-016-0520-4

[38] Liu Z, Song X, Tang Z. Integrating virtual samples and fuzzy discriminant analysis for sparse representation-based face classification. 
J. Electronic Imaging. 2015; 24 (2): 023013. https : //doi.org/ 10.1117/1.JEI.24.2.023013

[39] Tang B, Luo S, Huang H. High performance face recognition system by creating virtual sample. In: Proceedings of the International Conferenceon Neural Networks and Signal Processing. 2003: 972-5. https://doi.org/10.1109/icnnsp.2003.1280763

[40] Xu Y, Zhang Z, Lu G, et al. Approximately symmetrical face images for image preprocessing in face recognition and sparse representation based classification. Pattern Recognition. 2016; 54: 68-82. https://doi .org/10.1016/j.patcog. 2015.12.017

[41] Xu Y, Lu Y. Adaptive weighted fusion: A novel fusion approach for image classification. Neurocomputing. 2015; 168: 566-74. https : //doi.org/10.1016/j.neucom.2015.05.070

[42] Georghiades A, Belhumeur P, Kriegman D. From few to many: Illumination cone models for face recognition under variable lighting and pose. IEEE Trans. Pattern Anal. Mach. Intell. 2001; 23 (6): 643-60. https://doi.org/10.1109/34.927464

[43] Georgia Tech Face Database; 2007. available on: http://www . an efian.com/face_reco.html

[44] Li SZ, Lei Z, Ao M. The HFB face dataset for heterogeneous face biometrics research. In: Proceedings of the IEEE Computer Society Conference on Computer Vision and Pattern Recognition Workshops (CVPRW). 2009: 1-8.

[45] Belhumeur PN, Hespanha JP, Kriegman DJ. Eigenfaces vs. Fisherfaces: Recognition using class specific linear projection. IEEE Trans. Pattern Anal. Mach. Intell. 1997; 19 (7): 711-20. https: //doi.org/10.1109/34.598228

[46] Zhang T, Fang B, Yuan Y, et al. Multiscalefacial structure representation for face recognition under varying illumination. Pattern Recognition. 2009; 42(2): 252-8. https ://doi.org/10.1016/j. patcog.2008.03.017 